Uribe Macías, M.E. (2013). Modelo de gestión de la calidad en el servicio al cliente: propuesta para las grandes superficies. Revista Lebret (5). Bucaramanga, Colombia: Universidad Santo Tomás, pp 333 - 354.

\title{
Modelo de gestión de la calidad en el servicio al cliente: propuesta para las grandes superficies*
}

\section{Model of Quality Management in Customer Service: Proposal for Hypermarkets}

Mario Enrique Uribe Macías

\begin{abstract}
Resumen
En Colombia $40 \%$ de las compras de bienes básicos se hace en las grandes superficies. A pesar de que poseen certificaciones de calidad, día a día los clientes siguen presentando quejas. Se desarrolló un proyecto de investigación para determinar la percepción que tienen sus clientes con respecto a la calidad del servicio recibido, con resultados como la determinación de la importancia que para los clientes tienen aspectos tales como accesibilidad al parqueadero, amabilidad de los dependientes, calidad de los productos, precios, surtido, ubicación del supermercado y facilidades de pago. Con base en la percepción de los clientes y como aporte al desarrollo del tema, se propone un Modelo de gestión de la calidad del servicio al cliente para esas superficies.
\end{abstract}

\section{Palabras clave}

Servicio, Calidad, Sistema de Gestión de la Calidad, Grandes Superficies.

Código de Clasificación JEL: M19

\begin{abstract}
In Colombia, $40 \%$ of the basic goods are purchased in hypermarkets. Notwithstanding they have quality certifications, customers keep on complaining day after day. This project was developed in order to determine the perception customers have with respect to the quality of the service received, with results that determine things such as the importance customers give to parking accessibility, shop assistant friendliness, products quality, prices, assortment, supermarket location and payment facilities. Based on clients' perception and as a contribution to this topic development, a Customer service quality management model is proposed for these hypermarkets.
\end{abstract}

\section{Keywords}

Service, Quality, Quality Management System, Hypermarkets.

\footnotetext{
* El artículo presenta resultados del Proyecto de investigación "Análisis de la percepción de la calidad del servicio recibido y propuesta de un modelo de gestión de calidad en el servicio al cliente, orientado a las grandes superficies de la ciudad de Ibagué", financiado por el Fondo de Investigaciones de la Universidad del Tolima, Colombia.

1 Magíster en Administración, Tecnológico de Monterrey. Director grupo de investigación GIDEUT. Profesor Asociado de la Universidad del Tolima, Colombia.Correo electrónico: meuribem@ut.edu.co, meuribem@gmail. com.
} 


\section{Introducción}

El sector de los servicios se constituyó desde hace varias décadas en factor fundamental de desarrollo y crecimiento de los diferentes países, de tal manera que el cliente es (o debe ser) el punto focal de todas las decisiones y acciones de la organización de servicio.

Las tendencias de consumo en Colombia muestran que alrededor del $40 \%$ de las compras de bienes básicos (canasta familiar) se realizan en las llamadas Grandes Superficies. Las empresas de este sector han venido sufriendo cambios importantes durante los últimos años, desde su estructura de propiedad hasta su portafolio de servicios en búsqueda permanente de una mejor posición competitiva. No obstante lo anterior, son conocidas las permanentes quejas de los clientes respecto a la calidad de los servicios prestados por estos centros de venta.

De acuerdo con esta óptica, se desarrolló el proyecto de investigación "Análisis de la percepción de la calidad del servicio recibido y propuesta de un modelo de gestión de la calidad en el servicio al cliente, orientado a las grandes superficies de la ciudad de Ibagué", aprobado por el Comité Central de Investigaciones de la Universidad del Tolima proponiendo como su objetivo general la formulación de un modelo de gestión para la calidad en el servicio al cliente orientado a las grandes superficies.

Por la importancia del servicio al cliente, es importante medir su satisfacción, entendiendo que ésta evalúa el desempeño desde el punto de vista del consumidor y brinda una plataforma para la alineación estratégica de los recursos organizacionales, con el fin de entregar lo que es más importante para sus clientes, como lo plantea Pedic (2004).

El presente trabajo está encaminado a presentar el modelo diseñado para asegurar la satisfacción del cliente de las grandes superficies, en lo que a servicio se refiere, a partir del análisis de los resultados empíricos obtenidos mediante la aplicación de diferentes técnicas de recolección primaria y secundaria, y su confrontación con algunos modelos teóricos consultados.

\section{Planteamiento Teórico}

Para el proceso de formulación del anteproyecto y posteriormente para iniciar la investigación, se realizó una indagación exhaustiva tendiente a ubicar estudios que permitieran conocer el estado del arte en el tema. Los resultados fueron muy pobres, pues lo que se encuentra está orientado al tema de mercadeo pero no al de servicio y, mucho menos, a los sistemas de gestión de la calidad.

Esta situación fue corroborada en las entrevistas realizadas a los gerentes y/o administradores de las grandes superficies, quienes evidenciaron que realizan una serie de acciones encaminadas a mejorar la fidelidad de sus clientes, pero éstas 
no obedecen a un sistema intencionalmente diseñado, planificado y ejecutado. En general, podría afirmarse que estas acciones están planeadas como respuesta al alto nivel de rivalidad que existe en el sector, por lo cual son programas de fidelización que, si bien tocan el tema del servicio al cliente, son orientados más desde el enfoque de marketing que el del servicio.

En cuanto al soporte teórico de la investigación, éste se aborda desde tres referentes, particular cada uno de ellos pero todos relacionados entre sí: Servicio (Gerencia del Servicio), Calidad (Gerencia de la Calidad), y Gestión de la Calidad en el servicio (Sistemas de Gestión de la Calidad). Específicamente se indagó a los clientes con respecto al servicio recibido de parte de las grandes superficies, pero desde la óptica de la calidad percibida, con el objeto de diseñar y proponer un modelo de gestión de la calidad específico para el servicio al cliente, en este tipo de empresas.

\subsection{Servicio}

No obstante los diferentes puntos de vista con respecto al concepto de servicio, resulta necesario reconocer este concepto desde la teoría de la Gerencia del Servicio. El servicio es un conjunto de prestaciones adicionales al producto o servicio principal de la empresa que el cliente espera recibir, en contraprestación al precio que paga y a la reputación de la organización que lo presta. Significa este concepto que no es una referencia a la actividad principal de la empresa (por ejemplo, un servicio de salud, un servicio educativo, entre otros), sino a elementos adicionales que se convierten en diferenciadores en un mercado que es altamente competitivo, orientado a satisfacer las expectativas de los clientes con el fin de generar relaciones de largo plazo (fidelidad de clientes).

La exigencia competitiva de los mercados hace del servicio un elemento diferenciador que permite la generación de valor a las organizaciones que buscan la satisfacción del cliente y su fidelización, hoy no basta con ofrecer productos de calidad sino que el servicio se convierte en la ventaja competitiva por excelencia (Uribe, 2013). Kotler (2001) define el servicio como cualquier acto o desempeño que una parte puede ofrecer a otra, el cual es intangible y no da origen a la propiedad de algo. Con frecuencia los servicios acompañan los bienes, pero también cada vez con más frecuencia, desempeñan un papel importante en la economía global y bienestar de la comunidad.

La satisfacción del cliente se considera como el grado en que se cumplen sus expectativas con relación a un producto y el nivel en que dicho producto satisface sus necesidades dando como resultado una ventaja competitiva. La satisfacción del cliente es, a su vez, la percepción sobre el grado en que se cumplen los requisitos (Norma ISO 9000:2000). Identificar y analizar la percepción del cliente constituye, por tanto, un aspecto relevante en términos de la medición de la calidad del servicio para determinar el grado de satisfacción del cliente, entendiendo que éste evalúa el 
desempeño de la organización y brinda una plataforma para la alineación estratégica de los recursos organizacionales, con el fin de entregar lo que es más importante para sus clientes (Pedic, 2004).

\subsection{Calidad}

Mucho se ha discutido acerca del concepto de calidad y los términos que de él se derivan. El concepto de calidad es muy dinámico, depende de muchos factores $\mathrm{y}$ ha venido evolucionado permanentemente. Actualmente, se puede definir la calidad como el conjunto de características que posee un producto o servicio así como su capacidad de satisfacer los requerimientos del usuario; supone que el producto o servicio debe cumplir con las funciones y especificaciones para las que ha sido diseñado y que debe ajustarse a las necesidades expresadas por los clientes (Cuatrecasas, 2010). La calidad implica satisfacer las necesidades y deseos de los usuarios cumpliendo con los requerimientos del producto o servicio, superando las expectativas del cliente y haciendo desde la primera vez bien lo que haya que hacer.

La norma ISO 9000:2000 conceptúa que la calidad es el grado en el que un conjunto de características inherentes al producto cumple con las necesidades o expectativas establecidas. La gestión de la calidad total, como parte del concepto moderno de la calidad, comprende la forma de gestión de un organismo, centrado en la calidad y basado en la participación de todos sus miembros que apunta al éxito a largo plazo a través de la satisfacción del cliente, concepto que se relaciona con el aseguramiento de la calidad, el cual está orientado a proporcionar confianza en que se cumplirán los requisitos de la calidad.

La versión más reciente de la Norma Internacional ISO (9001:2008) recopila avances significativos en el manejo de los sistemas de gestión de la calidad. En esta norma, se considera que la satisfacción del cliente es una de las medidas de desempeño del sistema de gestión de la calidad, por lo cual la organización debe realizar seguimiento de la información relativa a la percepción del cliente. Este seguimiento puede incluir la obtención de elementos de entrada de fuentes como las encuestas de satisfacción del cliente, entre otras.

\subsection{Sistema de gestión de la calidad}

Implementar un sistema de gestión de la calidad en una organización es una decisión estratégica que implica cambios y beneficios orientados a mejorar continuamente el desempeño de la empresa tomando en cuenta las necesidades de todas las partes interesadas y que demuestra la capacidad que tiene la organización para cumplir los requerimientos del cliente y las leyes que aplican.

Este sistema constituye un conjunto de elementos mutuamente relacionados que interactúan para establecer la política, los objetivos de calidad y la forma de lograrlos (Norma ISO 9000:2000). El control de calidad orienta la organización al 
cumplimiento de los requisitos de la calidad y al cumplimiento de los objetivos, considerando el enfoque al cliente, el liderazgo y la participación del personal como algunos de los principios de la calidad.

Un sistema de gestión de una organización, según la Norma ISO 9000:2000, se define como un "Conjunto de elementos mutuamente relacionados o que interactúan para establecer la política y los objetivos y para lograr dichos objetivos"; por su parte, un sistema de gestión de la calidad es un "sistema de gestión para dirigir y controlar una organización con respecto a la calidad” (López, 2006 p.28).

Desarrollar un modelo de gestión de la calidad mediante la aplicación del enfoque basado en procesos, permite una interacción entre las distintas actividades que tenga la empresa, facilitando el control continuo sobre los procesos individuales y los vínculos que se den entre éstos. Por consiguiente, si dentro del sistema de gestión de la calidad se utiliza este enfoque, se enfatiza la importancia de: "la comprensión y el cumplimiento de los requisitos, la necesidad de considerar los procesos en términos que aporten valor, la obtención de resultados del desempeño y eficacia del proceso, y la mejora continua de los procesos con base en mediciones objetivas" (ISO, 2000, ii).

\section{Metodología}

El proyecto de investigación se realizó estudiando la totalidad de las grandes superficies existentes en Ibagué para la fecha del trabajo: Almacenes Éxito, Carrefour, Makro, Home Center, Mercacentro y Almacenes Yep, que son aquellas que tienen cinco o más cajas registradoras o puntos de pago.

En este trabajo se estudiaron tres poblaciones: la primera constituida por los clientes in situ que concurren a hacer sus compras en estas superficies; la segunda, conformada por los habitantes de sus áreas vecinas y la tercera, por los gerentes o administradores de estas empresas. En la Tabla 1 se especifica lo correspondiente.

Tabla 1. Recolección de información primaria por tipo de población

\begin{tabular}{ccc}
\hline POBLACIÓN & CENSO/MUESTRA & TIPO DE INSTRUMENTO \\
\hline Clientes in situ & $\begin{array}{c}\text { Muestreo no probabilístico por } \\
\text { conveniencia y juicio }\end{array}$ & $\begin{array}{c}\text { Cuestionario estructurado de objetivo } \\
\text { claro }\end{array}$ \\
\hline $\begin{array}{c}\text { Gerentes o } \\
\text { Habitantes vecinos }\end{array}$ & $\begin{array}{c}\text { Muestreo no probabilístico por } \\
\text { conveniencia y juicio }\end{array}$ & $\begin{array}{c}\text { Cuestionario estructurado de objetivo } \\
\text { claro }\end{array}$ \\
\hline Censo & Entrevista semi estructurada \\
\hline
\end{tabular}

Fuente: Autor. 
Para realizar el tratamiento y análisis de la información, se hizo uso de la estadística descriptiva que permitió conocer frecuencias, e igualmente la técnica de análisis multivariado, a través de la cual se utilizaron los métodos factorial y de k-medias con el propósito de agrupar variables en factores $\mathrm{y}$, posteriormente, realizar una interpretación global de las variables del estudio. En la investigación de tipo descriptiva explicativa se planteó inicialmente una estructura teórica que sustenta el desarrollo del trabajo, y sobre la que se seleccionaron las variables acerca de las cuales se recolectó la información relativa a dimensiones o componentes del fenómeno a investigar para someterlas a continuación a medición, evaluación y análisis, con el propósito de describir el comportamiento de la situación objeto de estudio. Posteriormente, se diseñaron los instrumentos de recolección de datos con las variables objeto de análisis, de acuerdo con lo ya planteado.

Una vez superados estos análisis y disponiendo de resultados concretos y confiables, se procedió al diseño del modelo de gestión de calidad en el servicio para las grandes superficies de la ciudad de Ibagué.

\section{Resultados}

\subsection{Variables que determinan la decisión de compra desde la perspectiva del cliente}

De la estructura teórica se desprenden una serie de variables que constituyen los elementos más sensibles que permiten medir la calidad en el servicio que reciben los consumidores, entendida ésta según Cuatrecasas (2010), como el conjunto de características que posee un producto o servicio, así como su capacidad de satisfacer los requerimientos del usuario.

Se realizó la selección de criterios para desarrollar la investigación y, en primera instancia, se determinó la importancia que para los clientes tienen aspectos tales como accesibilidad al parqueadero, amabilidad de los dependientes, calidad de los productos, precios, surtido, ubicación del supermercado y facilidades de pago, entre otros aspectos, en el momento de realizar sus compras. Igualmente, se indagó acerca de la evaluación que los clientes dan a estos mismos elementos con relación al servicio recibido.

De otra parte, se calificó el servicio que reciben los clientes por parte del personal de seguridad, impulsadores, mercaderistas, cajeros, empacadores, supervisores y personal de servicio al cliente. Igualmente se indagó sobre la calidad de la atención de las oficinas de servicio al cliente en cada uno de estos almacenes, y la eficiencia en los servicios de asesoría, garantía o postventa de los supermercados. En lo relacionado con los factores considerados por los clientes en el momento de la compra, se obtuvieron 
Modelo de gestión de la calidad en el servicio al cliente: propuesta para las grandes ...

más de 500 observaciones, dado que muchos clientes identificaron más de un motivo; éstos se agrupan en 15 categorías que se presentan en la Tabla 2 y la Figura 1.

Tabla 2. Motivos para seleccionar el lugar de compra

\begin{tabular}{|c|c|c|c|c|c|}
\hline & & tat $=9$ & hosens: & thesion & netants \\
\hline \multirow[t]{17}{*}{$1+200$} & 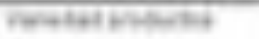 & $\mathrm{se}$ & 251 & 178 & -1 \\
\hline & 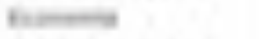 & th: & Int & 1114 & $\mathrm{x}=1$ \\
\hline & 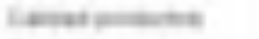 & $w$ & $\tan t$ & inat & $=1$ \\
\hline & henest & net & $=1$ & n= & vat \\
\hline & Romettent & 4 & El & 4.1 & $=1$ \\
\hline & 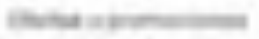 & $\mathbf{a}$ & wi & 14 & $\operatorname{man} i$ \\
\hline & 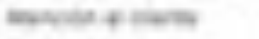 & $=$ & 21 & 13 & 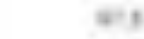 \\
\hline & Ateroute & t & 14 & 14 & $m i$ \\
\hline & Hentavianese & t & t & it & at \\
\hline & tenera & 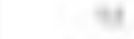 & ct & 14 & wi \\
\hline & $7 m$ & 4 & t & a & xil \\
\hline & 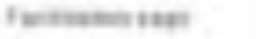 & 4 & $t$ & $a$ & mis \\
\hline & $=4=$ & s & 1 & t & 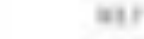 \\
\hline & 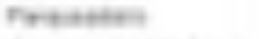 & 4 & 4 & a & wi 1 \\
\hline & 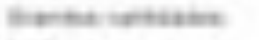 & $\mathbf{a}$ & 13 & 13 & $m:$ \\
\hline & venter & $a$ & $+s$ & ta & $7=4$ \\
\hline & $t w$ & ant & $=4$ & $1 \mathrm{kn}$ & \\
\hline Swnotest & thenent & t & 1 & & \\
\hline has & & ten & cast & & \\
\hline
\end{tabular}

Fuente: Autor.

Figura 1. Motivos por los cuales los clientes seleccionan su lugar de compras

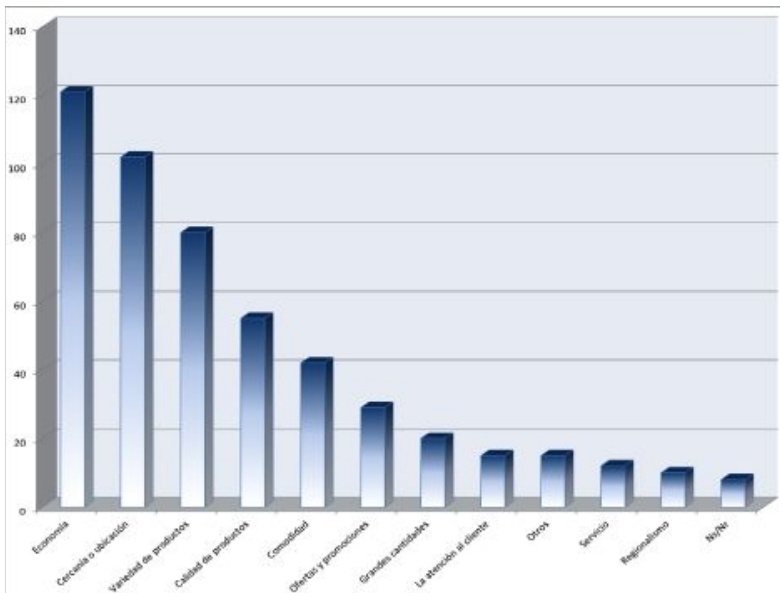

Fuente: Autor. 
De acuerdo con estos resultados, se puede decir que los principales motivos por los cuales los clientes escogen su sitio de compra son la economía, la cercanía, la variedad de productos y/o servicios y la percepción de calidad que tienen con respecto a los productos que allí se ofrecen, respuestas que representan el 70,6\% del total.

Se encuentra, de la misma manera, que los aspectos menos relevantes para los clientes al momento de escoger su sitio de compra son el horario, el parqueadero y el servicio de garantía (contenidos en el aspecto "Otros"). En este mismo sentido, al preguntarse a los miembros de la población vecina se encuentra que de, 177 respuestas, la cercanía (55\%), economía $(20 \%)$ y la variedad de productos $(20 \%)$ representan el $95 \%$ del total de las respuestas, lo cual coincide en gran medida con las respuestas entregadas por los clientes encuestados in situ; el 5\% adicional expresa que compran en otros lugares argumentando que los artículos son costosos, que no guardan fidelidad por algún almacén en particular y el regionalismo, entre los aspectos considerados. La situación se puede analizar en la Figura 2.

Figura 2. Motivos de selección del sitio de compra por la población vecina

\section{Si la respuesta es positiva, ¿por qué compra en este almacén?}

\section{ECercania Economia = Variedad de productos $=$ Otros}

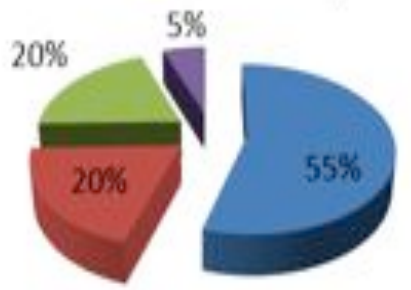

Fuente: Autor.

\subsection{Importancia concedida por los clientes a las variables relacionadas con el servicio}

Paralelamente a los factores considerados por los clientes de estas superficies al momento de realizar sus compras, es necesario priorizar la importancia de cada uno de ellos con el propósito de establecer aquéllos que son críticos y que definen su decisión final de compra.

En términos generales, las variables incluidas en el cuestionario estructurado de objetivo claro aplicado a los 380 clientes in situ, fueron calificadas en promedio 
por encima del $90 \%$ en las categorías muy importante e importante. Así, el factor relacionado con la calidad de los productos obtuvo un $99,2 \%$, amabilidad del personal $98,1 \%$, atención al cliente 97,4\%, surtido 96,8\%, economía (precio) 95,8\%, agilidad en la atención 95,6\%, claridad en la información y amplitud de los pasillos 95,5\%, ofertas y promociones $92,9 \%$, señalización y garantía y servicio postventa $92,1 \%$, horario de atención $90,5 \%$, cercanía a la superficie $89,7 \%$, facilidades de pago $87,7 \%$, publicidad 79,8, accesibilidad al parqueadero 78,7\% y servicios adicionales $72,3 \%$. Esta situación se presenta en la Tabla 3.

Estas valoraciones coinciden con los aspectos que fueron considerados por los usuarios en la selección del sitio de compra lo que, en principio, indicaría una atención primordial por parte de los directivos de estas superficies a estas cuestiones en particular.

De la misma manera, es conveniente señalar aquellas variables que en alguna proporción son calificadas como no importantes, siendo en este caso las más representativas la publicidad $(19,5 \%)$, la accesibilidad al parqueadero $(17,1 \%)$, facilidades de pago (12,1\%), cercanía (10,3\%) y horario de atención (9,5\%). Igualmente hay que destacar que el $20,5 \%$ y el $4,2 \%$ de los encuestados no responden las preguntas relacionadas con servicios adicionales y accesibilidad al parqueadero.

Tabla 3. Nivel de importancia de las variables de servicio

\begin{tabular}{lcccccccc}
\hline \multicolumn{1}{c}{ VARIABLES } & $\begin{array}{c}\text { NO/ } \\
\text { IMP }\end{array}$ & $\mathbf{\%}$ & $\begin{array}{c}\text { ES } \\
\text { IMPORTANTE }\end{array}$ & $\mathbf{\%}$ & $\begin{array}{c}\text { MUY } \\
\text { IMPORTANTE }\end{array}$ & $\mathbf{\%} / \mathbf{N R}$ & $\%$ \\
\hline Acceso al parqueadero & 65 & 17 & 160 & 42 & 139 & 37 & 16 & 4,2 \\
\hline Amabilidad del personal & 7 & 1,8 & 111 & 29 & 262 & 69 & 0 & 0 \\
\hline Amplitud de los pasillos & 17 & 4,5 & 170 & 45 & 193 & 51 & 0 & 0 \\
\hline Señalización & 30 & 7,9 & 158 & 42 & 192 & 51 & 0 & 0 \\
\hline Atención al cliente & 10 & 2,6 & 112 & 30 & 258 & 68 & 0 & 0 \\
\hline Calidad de los productos & 3 & 0,8 & 112 & 30 & 265 & 70 & 0 & 0 \\
\hline Economía/precio & 13 & 3,4 & 123 & 32 & 241 & 63 & 3 & 0,8 \\
\hline Surtido & 11 & 2,9 & 140 & 37 & 228 & 60 & 1 & 0,3 \\
\hline Agilidad en la atención & 17 & 4,5 & 115 & 30 & 248 & 65 & 0 & 0 \\
\hline Facilidades de pago & 46 & 12 & 164 & 43 & 169 & 45 & 1 & 0,3 \\
\hline Cercanía & 39 & 10 & 160 & 42 & 181 & 48 & 0 & 0 \\
\hline Horario de atención & 36 & 9,5 & 166 & 44 & 178 & 47 & 0 & 0 \\
\hline Ofertas/promociones & 27 & 7,1 & 150 & 40 & 203 & 53 & 0 & 0 \\
\hline Garantía y servicio postventa & 25 & 6,6 & 140 & 37 & 210 & 55 & 0 & 0 \\
\hline Claridad de la información & 17 & 4,5 & 157 & 41 & 206 & 54 & 0 & 0 \\
\hline Publicidad & 74 & 20 & 161 & 42 & 142 & 37 & 3 & 0,8 \\
\hline Servicios adicionales & 27 & 7,1 & 148 & 39 & 127 & 33 & 78 & 21 \\
\hline
\end{tabular}

Fuente: Autor. 


\subsection{Nivel de satisfacción de los clientes con relación a los servicios recibidos}

En desarrollo del proceso de investigación se procedió a evaluar el grado de satisfacción expresado por los clientes con relación a esos criterios de desempeño. Se inicia por destacar las variables que presentan unas evaluaciones regulares y deficientes por parte de los clientes en lo que al nivel de satisfacción se refiere, siendo ellas: agilidad en la atención $(27,4 \%)$, servicios adicionales $(22,7 \%)$, atención al cliente $(15,8 \%)$, garantía y servicio postventa $(14,5 \%)$, precios $(14,2 \%)$, ofertas y promociones $(13,9 \%)$, amabilidad del personal $(13,2 \%)$, claridad en la información $(12,1 \%)$, amplitud de los pasillos (11,6\%) y publicidad (11\%). En la siguiente Tabla se observa en detalle esta situación.

Tabla 4. Nivel de satisfacción de los clientes

\begin{tabular}{lcccccccccc}
\hline \multicolumn{1}{c}{ VARIABLES } & EXC & $\%$ & BUENO & $\mathbf{\%}$ & REGUL & $\mathbf{\%}$ & DEFIC & $\%$ & NS/NR & $\%$ \\
\hline Acceso al parqueadero & 83 & 22 & 221 & 58 & 22 & 5,8 & 2 & 1 & 51 & 14 \\
\hline Amabilidad del personal & 90 & 24 & 240 & 63 & 47 & 12 & 3 & 1 & 0 & 0 \\
\hline Amplitud de los pasillos & 92 & 24 & 242 & 64 & 42 & 11 & 2 & 1 & 2 & 0,5 \\
\hline Señalización & 69 & 18 & 267 & 70 & 30 & 7,9 & 14 & 4 & 0 & 0 \\
\hline Atención al cliente & 91 & 24 & 229 & 60 & 48 & 13 & 12 & 3 & 0 & 0 \\
\hline Calidad de los productos & 128 & 34 & 238 & 63 & 13 & 3,4 & 1 & 0 & 0 & 0 \\
\hline Economía/precio & 74 & 20 & 252 & 66 & 52 & 14 & 2 & 1 & 0 & 0 \\
\hline Surtido & 88 & 23 & 264 & 70 & 28 & 7,4 & 0 & 0 & 0 & 0 \\
\hline Agilidad en la atención & 50 & 13 & 226 & 60 & 87 & 23 & 17 & 5 & 0 & 0 \\
\hline Facilidades de pago & 96 & 25 & 241 & 63 & 28 & 7,4 & 7 & 2 & 8 & 2,1 \\
\hline Cercanía & 152 & 40 & 191 & 50 & 32 & 8,4 & 3 & 1 & 2 & 0,6 \\
\hline Horario de atención & 135 & 36 & 227 & 60 & 16 & 4,2 & 0 & 0 & 2 & 0,6 \\
\hline Ofertas/promociones & 102 & 27 & 219 & 58 & 51 & 13 & 2 & 1 & 6 & 1,6 \\
\hline Garantía y servicio postventa & 65 & 17 & 231 & 61 & 44 & 12 & 11 & 3 & 29 & 7,6 \\
\hline Claridad de la información & 67 & 18 & 254 & 67 & 36 & 9,5 & 10 & 3 & 13 & 3,4 \\
\hline Publicidad & 80 & 21 & 245 & 65 & 35 & 9,2 & 7 & 2 & 13 & 3,4 \\
\hline Servicios adicionales & 28 & 7,4 & 149 & 39 & 82 & 22 & 4 & 1 & 117 & 31 \\
\hline & & & & & & & & & 0 \\
\hline
\end{tabular}

Fuente: Autor.

Del total de las variables consideradas importantes y muy importantes por los clientes de estos almacenes, el 56,25\% no son bien calificadas por ellos. Igualmente de las 109 personas encuestadas de la población vecina que dicen ser clientes de estas superficies, el 87\% manifiestan sentirse satisfechos al realizar las compras en estas superficies; el 13\% adicional, que dice no sentirse satisfecho, afirma que las causas de su inconformidad son la deficiente atención al cliente, demoras en las cajas, precios altos, problemas de información e inexistencia de producto. Como puede observarse, 
la mayoría de estos aspectos coinciden con los criterios que también reciben las más bajas calificaciones por parte de los clientes in situ. Esta situación se aprecia en la Figura 3.

Figura 3. Nivel de satisfacción de la población vecina

\section{¿Se siente satisfecho al realizar compras en este almacén?}

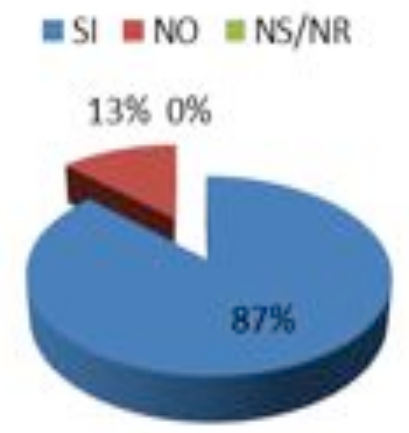

Fuente: Autor.

Si a todo lo anterior se suma que las variables servicios adicionales, accesibilidad al parqueadero y garantía y servicio postventa, en unos porcentajes del $30,8 \%, 13,5 \%$ y $7,6 \%$ no reciben respuesta por parte de los usuarios, queda la sensación que en materia de calidad del servicio ofrecido por estas superficies aún falta mucho por hacer. Como se puntualizó anteriormente, en el análisis a los servicios adicionales, existe gran inconformidad en la prestación de éstos; si al porcentaje del 30,8\% de usuarios que no contestan, se le sumase el $22,7 \%$ de las personas que califican este aspecto como regular y deficiente, se tendría un porcentaje del 53,5\% de clientes que no aprecian el suministro de estos servicios tal como se están dando en el momento, máxime si se recuerda que el $72,3 \%$ de los clientes califican esta variable como importante y muy importante.

En lo que respecta a la accesibilidad al parqueadero puede argumentarse que quienes no responden son personas que no poseen vehículo y por lo tanto, no han utilizado el servicio. Con relación a la garantía y servicio postventa, este hecho se corrobora en la medida que siendo una variable a la cual los clientes le dan el $92,1 \%$ de importancia, el índice de insatisfacción entendido éste como la sumatoria de los porcentajes de las categorías regular y deficiente llega al 15\% que se considera muy significativo, atendiendo al hecho de que esta variable se constituye en uno de los pilares de la satisfacción del cliente. 
Figura 4. Evaluación de servicios adicionales

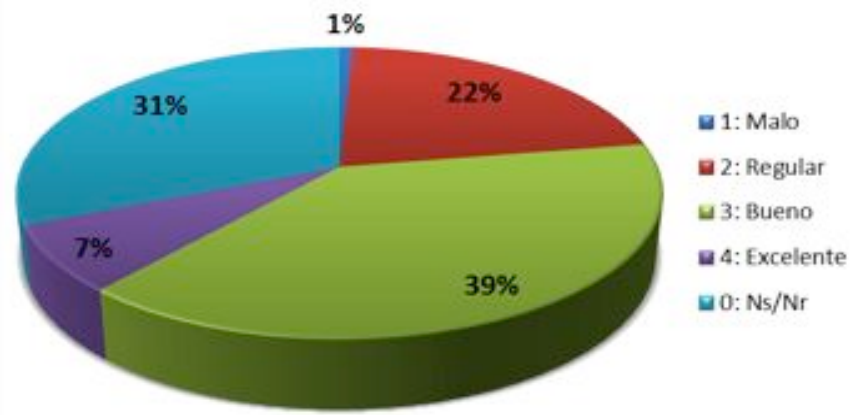

Fuente: Autor.

\subsection{El modelo de gestión de la calidad en el servicio}

Un sistema de gestión de la calidad es un conjunto de procesos, procedimientos y decisiones directivas, que contribuyen significativamente para que una empresa desarrolle su misión centrada en el cliente y orientada a lograr su plena satisfacción. En este sentido, el sistema de gestión es en primera instancia genérico, pues debe ser útil para un conjunto de empresas que tienen en sí mismas sus particularidades, pero debe tener un buen nivel de flexibilidad para que cada empresa, vista en sí misma, pueda adoptarlo y adaptarlo a sus propias realidades.

El modelo de gestión para la calidad en el servicio al cliente propuesto está basado en el enfoque de procesos aconsejado por la Norma ISO 9000, en cuanto a los sistemas de gestión de la calidad para las organizaciones pero no debe entenderse como una aplicación de esta norma. El enfoque de procesos es una mirada sistémica de la organización orientada hacia el cliente (su satisfacción), a partir del conocimiento y entendimiento de sus requisitos, los cuales generan la información de entrada que pone en movimiento, de manera articulada, los diferentes componentes (procesos) sugeridos.

El modelo está centrado en el desarrollo armónico de la gestión de la infraestructura, la prestación del servicio, el monitoreo y mejora continua, teniendo como eje articulador el talento humano de la organización, debidamente alineado con el direccionamiento estratégico que el nivel gerencial le imprime a toda la empresa. Se observa en la Figura 5.

Los modelos de gestión de la calidad establecen el "qué" en términos generales; es responsabilidad de cada organización responder a la pregunta de "cómo", considerando su propia realidad interna y la relación con su entorno. Esta propuesta se centra en el modelo y no pretende ser una guía para su implementación operativa. 


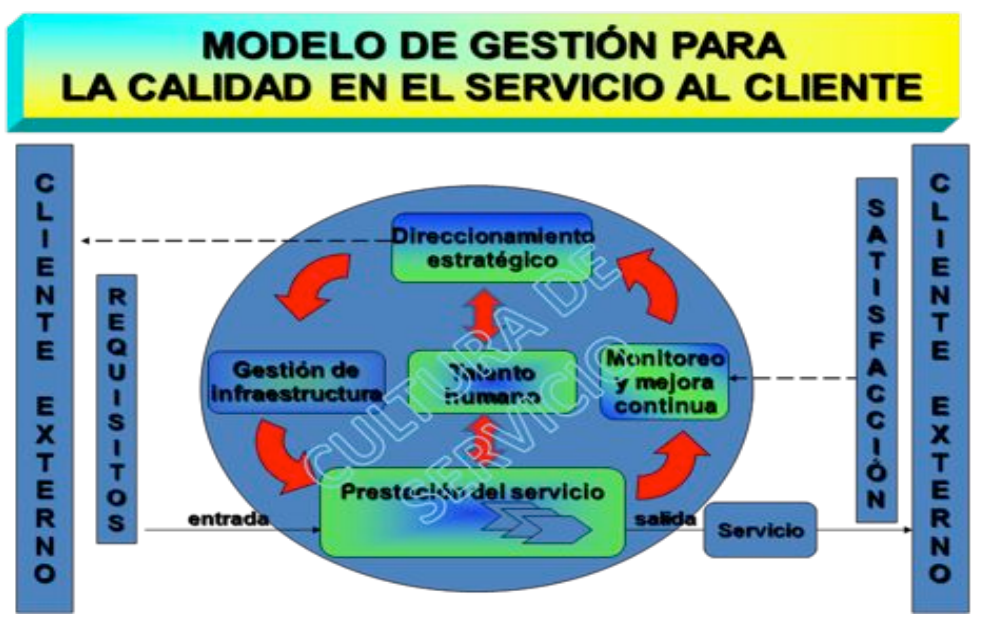

Fuente: Autor.

\subsubsection{Direccionamiento estratégico.}

Corresponde a todas las decisiones y actividades del nivel gerencial de la organización, orientadas a proyectar a la empresa en el largo plazo y a generar ventaja competitiva sustentable. Incluye el establecimiento de los grandes propósitos (la visión, la misión y los objetivos corporativos), la formulación de las políticas, la definición de la estrategia corporativa, el diseño de los planes de acción, la generación de una cultura organizacional (que se profundiza más adelante) y el sistema de control estratégico. Éste es un proceso que puede presentar variaciones de forma y algunas de fondo, dependiendo de la empresa y de los referentes teóricos que se utilicen. Este proceso puede apreciarse en la Figura 6.

Para efectos del modelo de gestión orientado a la calidad en el servicio al cliente, deberá formularse la estrategia del servicio, elemento diferenciador que en términos de Uribe (2013, p.105) "es considerada como una idea unificadora que orienta la atención de la gente de la empresa hacia las prioridades reales del cliente y focaliza a toda la organización hacia ellas".

Hace parte del direccionamiento estratégico la creación, mantenimiento y mejoramiento del sistema gerencial el cual, según Uribe (2011, p.111) “está conformado por todo el equipo directivo de la organización. Pero no sólo tiene que ver con las personas sino con sus actuaciones y la infraestructura que apoya al sistema para su funcionamiento". 
De igual manera, es responsabilidad de la dirección estratégica de la organización establecer y mejorar el sistema de reglas y regulaciones para el cliente. Este sistema, de acuerdo con el autor precitado "establece las condiciones por medio de las cuales el cliente interactúa con la empresa para la obtención de los diferentes servicios que demanda de ella. Al igual que en el caso del sistema gerencial, éste debe ser amable para el cliente, debe ser diseñado pensando en su facilidad y satisfacción" (Uribe, 2011 p. 112).

Figura 6. Esquema general de direccionamiento estratégico

\section{DIRECCIONAMIENTO ESTRATÉGICO}

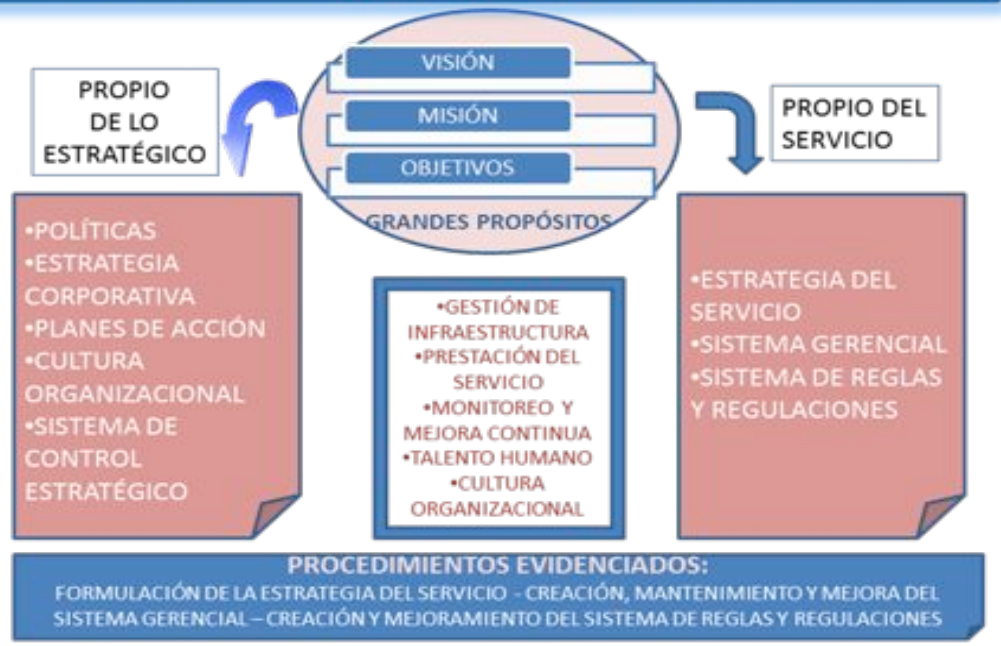

Fuente: Autor.

Para este proceso se deben evidenciar los procedimientos para la formulación de la estrategia del servicio, para la creación, mantenimiento y mejoramiento del sistema gerencial y para la creación y mejoramiento del sistema de reglas y regulaciones.

\subsubsection{Gestión de la infraestructura.}

Especial importancia para el caso en estudio representa la infraestructura: para los clientes es una variable fundamental para su satisfacción, a veces tan importante como la calidad de los productos que adquieren. En la infraestructura están contemplados los espacios físicos en los cuales se mueve y adelanta su actividad de compra el cliente: parqueadero, acceso y salida, pasillos, instalaciones, aire acondicionado y demás comodidades que le agregan valor a la experiencia del consumidor. 
La responsabilidad del adecuado suministro, mantenimiento y mejoramiento de la infraestructura en la empresa corresponde a su sistema gerencial. Debe asignar los recursos financieros requeridos para desarrollar las acciones mencionadas y establecer sistemas de información que les permita conocer en tiempo real las condiciones de la infraestructura y las opiniones de los clientes al respecto. En la Figura 7 se detallan estos componentes.

Es necesario crear, mantener y mejorar el sistema técnico de la empresa, entendido por Uribe (2011, p.113) como "todos los elementos que interactúan para producir el servicio". Al igual que los demás sistemas de la organización, éste debe ser amable con el cliente y facilitarle su relacionamiento con la empresa, máxime que a través del sistema técnico el cliente hace uso de los servicios ofrecidos por la organización.

Figura 7. Esquema general de gestión de la infraestructura

\section{GESTIÓN DE LA INFRAESTRUCTURA}

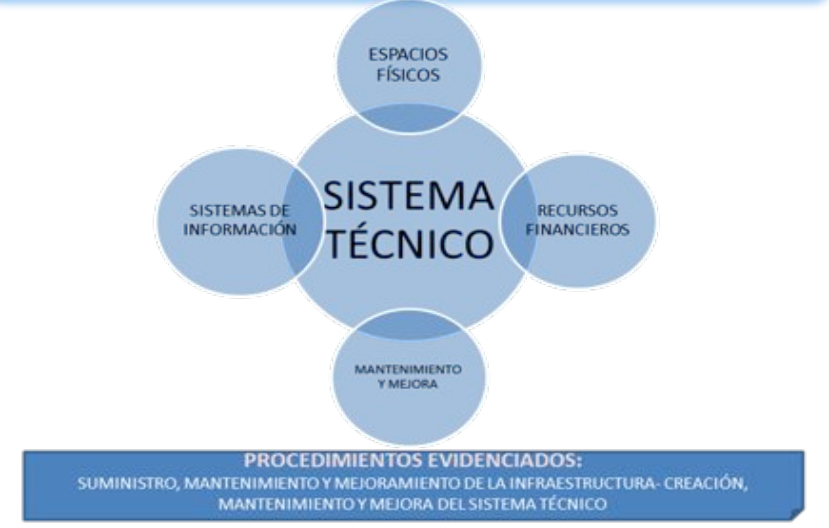

Fuente: Autor.

El sistema técnico incluye los procedimientos para prestar el servicio (profundizados en el siguiente ítem), los elementos físicos que apoyan la prestación del servicio y las personas que lo desarrollan y prestan. Para este proceso se deben evidenciar los procedimientos para el suministro, mantenimiento y mejoramiento de la infraestructura y para la creación, mantenimiento y mejoramiento del sistema técnico. 


\subsubsection{Prestación del servicio.}

La maximización de la experiencia del cliente se da cuando éste recibe los servicios que la empresa le ofrece. Para que los momentos de verdad sean agradables para el cliente, la empresa debe estandarizar los procedimientos orientados a la prestación (producción) del servicio.

Como consecuencia, se debe diseñar, mantener y mejorar el sistema del servicio de la empresa, basado en los requisitos del cliente, pero a su vez como resultado de un encadenamiento virtuoso: la declaración de la estrategia del servicio, el diseño del paquete de servicios y, finalmente, la prestación mediante la utilización del sistema del servicio que es considerado como el conjunto de mecanismos con los que se cuenta para satisfacer las necesidades del cliente.

Es necesario en este proceso evidenciar los procedimientos para la prestación de los diferentes servicios que la empresa les presta a sus clientes y para la gestión de las no conformidades del servicio. En la Figura 8 se sintetiza este proceso.

Figura 8. Esquema general de prestación del servicio

\section{PRESTACIÓN DEL SERVICIO}

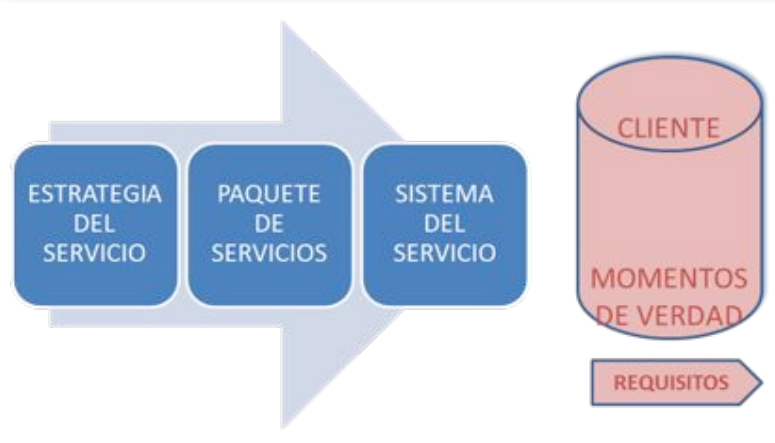

PROCEDIMIENTOS EVIDENCIADOS:

PRESTACIÓN DEL LOS SERVICIOS- GESTIÓN DE LAS NO CONFORMIDADES DEL SERVICIO

Fuente: Autor.

\subsubsection{Monitoreo y mejora continua}

Una de las responsabilidades inherentes a cualquier sistema de gestión de la calidad es su mejora continua y permanente con el objeto de que no pierda su vigencia, con mayor razón si se trata de un sistema orientado a la calidad del servicio al cliente, 
pues éste es una criatura inconstante que cambia sus expectativas y tendencias de consumo sin previo aviso. Para poder ser exitosa en esta tarea, la organización debe generar los espacios y mecanismos para realizar un monitoreo permanente a sus clientes y a su sistema. Reviste especial importancia la gestión del sistema PQR (peticiones, quejas y reclamos) de los clientes, que ofrece información importante, de primera mano, que le permite a la empresa realizar acciones de mejora.

Como herramienta fundamental para el desarrollo del monitoreo y fuente para el mejoramiento, la empresa debe generar un sistema de indicadores de gestión que le permita tomar decisiones para la mejora. Para este proceso se deben evidenciar los procedimientos para el desarrollo de las acciones de monitoreo; para el establecimiento, mantenimiento y mejoramiento de los indicadores de gestión; para el manejo de las peticiones, quejas y reclamos del cliente y para el desarrollo de las acciones de mejora continua. Se puede observar en la Figura 9.

Figura 9. Esquema general de monitoreo y mejora continua

\section{MONITOREO Y MEJORA CONTINUA}

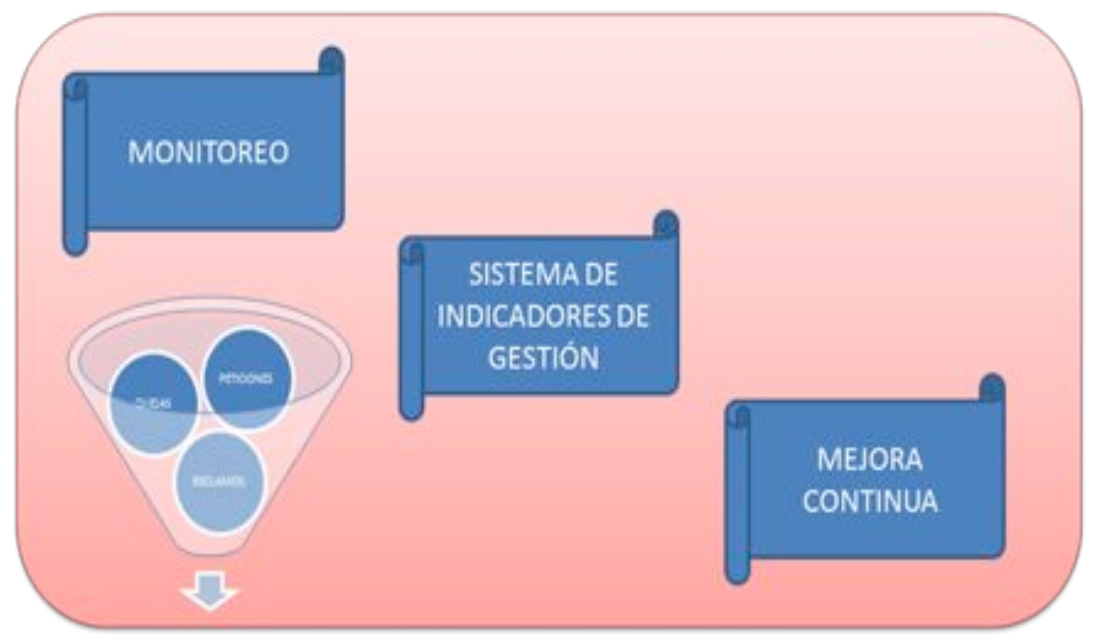

PROCEDIMIENTOS EVIDENCIADOS:

DESARROLLO DE UAS ACCIONES DE MONITOREO - ESTABIECIMIENTO, MANTENMIENTO Y MEJORAMIENTO DE LOS INOICADORES DE GESTION - MANEIO OE LAS PETICONES, OUEIAS Y RECLAMOS DEL CLIENTE - DESARROLLIO DE ACCIONES DE MEIORA CONTINUA

Fuente: Autor. 


\subsubsection{Talento humano}

Las personas son fundamentales para el desarrollo de las actividades empresariales; sobre ellas recae la responsabilidad de cumplir la promesa de servicio al cliente, desarrollar los procesos y procedimientos, utilizar la infraestructura, prestar los servicios, realizar el monitoreo y la mejora continua y, en fin, lograr que la organización viva.

En la perspectiva de Uribe "para asegurar que la empresa cuente con un talento humano competente para satisfacer al cliente debe iniciarse con un adecuado proceso de selección de personal, lo cual exige diseñar de manera acertada los perfiles de cargo para cada uno de los cargos de la organización” (Uribe, 2011, p. 115). En segunda instancia, la organización debe emprender procesos de sensibilización y capacitación permanente con su talento humano, al menos en dos sentidos: uno, entender y aplicar los diferentes elementos considerados en este modelo de gestión propuesto y dos, generar procesos de cambio que conduzcan a la transformación de la cultura organizacional existente a la deseada. Entonces, se debe formular y construir, de ser necesario, el conjunto de principios y valores, enfocados al cliente, que deben ejercitar todos los miembros de la organización.

Para este proceso se deben evidenciar los procedimientos para el diseño de los perfiles de cargo, para el proceso de selección del talento humano y para el diseño de la capacitación al personal, de acuerdo con el esquema general de la Figura 10.

Figura 10. Esquema general de talento humano

\section{TALENTO HUMANO}

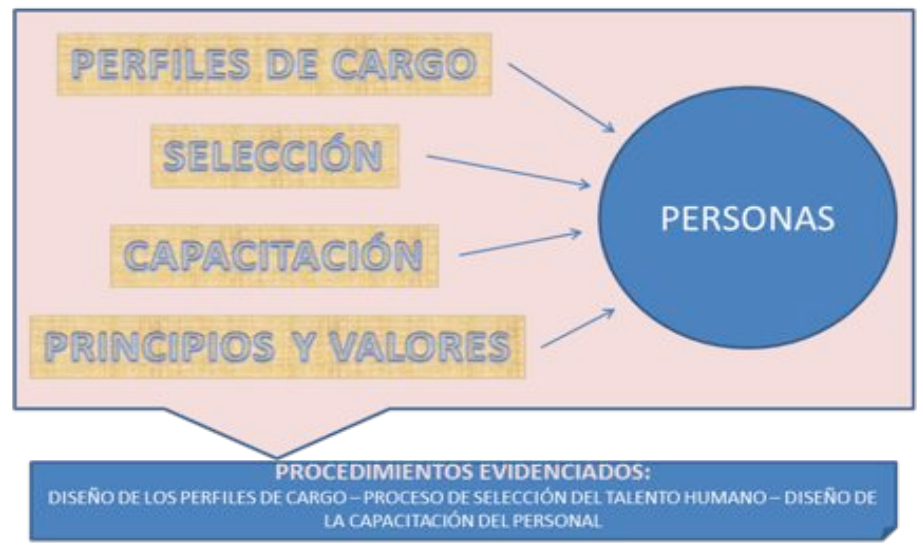

Fuente: Autor. 


\subsubsection{Cultura organizacional.}

Siendo la cultura organizacional "el modo de vida propio que cada organización desarrolla en sus miembros" (Chiavenato, 1995, citado por Reinoso y Uribe, 2009 p. 11) y "un sistema de significados compartidos dentro de una organización" (Robbins, 1996, citado por Reinoso y Uribe, 2009, p.12) resulta imprescindible para una organización orientada hacia el cliente, que implementa un modelo de gestión de la calidad para el servicio al cliente, lograr que la empresa (entendida como todos sus miembros) viva y comparta significados alrededor del precepto de que "el cliente es lo primero".

En este proceso se incorpora el conjunto de principios y valores que se definieron en el proceso anteriormente descrito, y se emprenden las acciones orientadas a lograr la transformación cultural. Ésta se logra mediante la utilización de acciones educativas, de sensibilización y de capacitación y de la intervención del direccionamiento estratégico (ítem 3.2.1) en la interpretación de dichos significados comunes, el establecimiento de las normas organizacionales y el ejercicio del ejemplo como factor de cambio.

Los anteriores procesos, base fundamental del modelo propuesto, deben convertirse en elementos culturales dentro de la organización; en otros términos, deben corresponder a la definición axiológica de la empresa (conjunto de principios y valores), a los hábitos que se ejercen, a las costumbres que se arraigan, a los ritos que se privilegian, a la estructura normativa de la organización que es compartida por todos sus miembros.

Figura 11. Esquema general de cultura organizacional

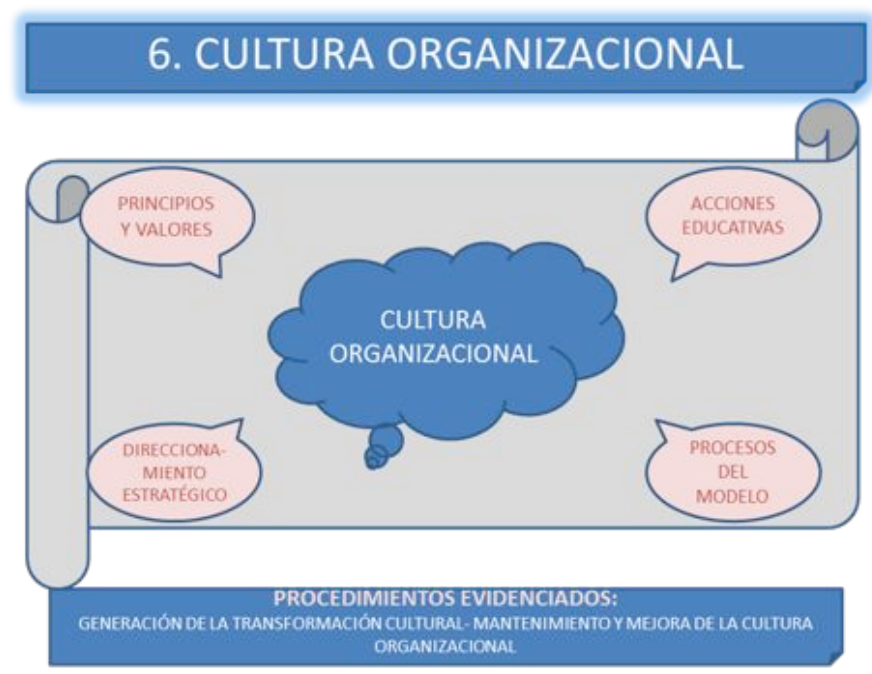

Fuente: Autor. 
Las evidencias de los procesos anteriores, en particular cada una de ellas, contribuyen a la generación de la cultura organizacional propicia para la ejecución del modelo. En este proceso debe evidenciarse, particularmente, el procedimiento para la generación de la transformación cultural y el mantenimiento y mejora de la cultura organizacional de la empresa.

\section{Conclusiones}

Existen diferentes referentes teóricos para abordar el concepto de servicio; en esta investigación se ha tomado como guía el siguiente: el servicio es un conjunto de prestaciones, adicionales al producto o servicio principal de la empresa, que el cliente espera recibir, en contraprestación al precio que paga y a la reputación de la organización que lo presta.

De igual forma, sucede con el concepto de calidad, la investigación ha tomado como referente el criterio que concibe la calidad como el grado en el que un conjunto de características inherentes al producto, cumple con las necesidades o expectativas establecidas. La gestión de la calidad total, como parte del concepto moderno de la calidad, comprende la forma de gestión de un organismo, centrado en la calidad y basado en la participación de todos sus miembros, que apunta al éxito a largo plazo a través de la satisfacción del cliente.

A través de esta investigación se identificaron 15 variables que, desde la perspectiva del cliente, determinan la calidad en el servicio en las grandes superficies de la ciudad de Ibagué, entre las cuales se encuentran la accesibilidad al parqueadero, la amabilidad del personal, la calidad de los productos, el precio, el surtido, la ubicación, las facilidades de pago, la amplitud de los pasillos, la orientación y señalización, la atención al cliente, la oferta de productos y servicios, la agilidad en la atención, la cercanía, el horario de atención, las ofertas y promociones, la garantía y servicio posventa, la publicidad y los servicios adicionales.

Se observó que según los clientes encuestados, los aspectos más importantes en su orden son: la agilidad en la atención, la accesibilidad al parqueadero y la atención al cliente, mientras que aspectos como la calidad de los productos, la amplitud de los pasillos y el horario de atención, son los aspectos calificados con menor importancia para ellos.

Los motivos principales que llevan a los clientes a escoger uno de los lugares de compra son la economía, la cercanía, la variedad de productos o servicios, y la calidad percibida de los productos ofrecidos; lo cual representa el $70.6 \%$ del total.

La mayoría de los usuarios $(27.79 \%$ del total de aspectos calificados como excelente) se encuentran satisfechos en aspectos como el horario de atención, la calidad de los productos y la ubicación del sitio de compra, pero muchos se encuentran insatisfechos con la agilidad en la atención, la garantía y el servicio postventa, la 
orientación y señalización y la atención al cliente $(56.67 \%$ del total de aspectos calificados como malo).

Al evaluar al personal de servicios y ponderar los resultados se determinó que el nivel de satisfacción de los clientes (calificación de excelente y bueno) es del $66.1 \%$. Por lo anterior, el servicio al cliente no se percibe como una ventaja competitiva en las grandes superficies de la ciudad de Ibagué, debido a las constantes quejas de usuarios insatisfechos.

En general, se concluye que el nivel de satisfacción de los clientes con respecto a los servicios que reciben en las grandes superficies es bueno, pero existen aspectos a mejorar principalmente en relación al personal que tiene contacto con los clientes, los servicios adicionales y los servicios de asesorías, garantías y postventa, durante los cuales se debe dar un mejor manejo a la información.

En cuanto a los planteamientos de los directivos de las grandes superficies, se encuentra que en general no se hace evidente un modelo de servicio al cliente que desarrolle cada organización; lo que se plantea son actividades y acciones puntuales, muy específicas que, aunque importantes, no son la esencia de un modelo y filosofía de trabajo orientados al cliente.

Con relación a la población vecina, éstos se encuentran satisfechos con la ubicación de las grandes superficies cerca de sus lugares de habitación, en términos de la importancia de la variable cercanía; además, porque manifiestan que sus predios se han valorizado, pero algunos manifiestan perjuicios, en particular por el cargue y descargue de mercancía en los almacenes, lo cual genera congestión vehicular, inseguridad y encarecimiento del costo de vida pues se generan algunos cinturones comerciales alrededor y/o por el incremento de los cánones de arrendamiento.

El modelo de gestión para la calidad en el servicio al cliente propuesto por la investigación está centrado en el desarrollo armónico de la gestión de la infraestructura, la prestación del servicio, el monitoreo y mejora continua, teniendo como eje articulador el talento humano de la organización, debidamente alineado con el direccionamiento estratégico que el nivel gerencial le imprime a toda la empresa.

\section{Referencias}

Albrecht, K. (1998). La revolución del servicio. Bogotá, Colombia: Legis Editores.

Albrecht, K; Zemke, R. (1991). Gerencia del Servicio. Bogotá, Colombia: Legis Editores.

Anzola, O. (2003). Una mirada a la cultura corporativa. Bogotá, Colombia: Universidad Externado de Colombia.

Cuatrecasas, L. (2010). Gestión integral de la calidad. Editorial Profit. 
Icontec. (2001). ISO 9000:2000. Guía para las pequeñas empresas. Bogotá, Colombia: Icontec.

Icontec. (2009). NTC-ISO 9001:2008. Sistemas de gestión de la calidad. Requisitos. Bogotá, Colombia: Icontec.

Johnson, G; Scholes, K. (2001). Direccion estratégica. Madrid, España: Pearson Educación.

López, Francisco. (2006). ISO 9000 y la planificación de la calidad. Bogotá, Colombia: Icontec.

Uribe, M y Reinoso J. F. ( 2009). Sistema de Indicadores de Gestión Bogotá, Colombia: Ediciones de la U

Uribe, M. (2011). Los sistemas de gestión de la calidad, el enfoque teórico y la aplicación práctica, Ibagué, Colombia: Editorial Universidad del Tolima.

Uribe, M. (2013). Gerencia del servicio. Alternativa para la competitividad. (2 ${ }^{\mathrm{a}}$ ed.) Bogotá, Colombia: Ediciones la U. 